\title{
(- OPEN ACCESS \\ Do patients who die from an alcohol-related condition 'drift' into areas of greater deprivation? Alcohol-related mortality and health selection theory in Scotland
}

\author{
Andrew Pulford, ${ }_{1}^{1}$ Ruth Gordon, ${ }^{2}$ Lesley Graham, ${ }^{2}$ James Lewsey, ${ }^{3}$ Gerry McCartney, \\ Mark Robinson $^{1}$
}

${ }^{1}$ Public Health Observatory, NHS Health Scotland, Glasgow, UK ${ }^{2}$ Information Services Division, NHS National Services Scotland, Edinburgh, UK ${ }^{3}$ Health Economics and Health Technology Assessment, Institute of Health \& Wellbeing, University of Glasgow, Glasgow, UK

Correspondence to Andrew Pulford, Public Health Observatory, NHS Health Scotland, Glasgow, G2 6QE, UK; andrew.pulford@nhs.net

Received 28 July 2017 Revised 8 November 2017 Accepted 21 November 2017 Published Online First 7 December 2017

\section{ABSTRACT}

Background Health selection has been proposed to explain the patterning of alcohol-related mortality by area deprivation. This study investigated whether persons who die from alcohol-related conditions are more likely to experience social drift than those who die from other causes.

Methods Deaths recorded in Scotland $(2013, \geq 21$ years) were coded as 'alcohol-related' or 'other' and by deprivation decile of residence at death. Acute hospital admissions data from 1996 to 2012 were used to provide premortality deprivation data. $\chi^{2}$ tests estimated the difference between observed and expected alcohol-related deaths by first Scottish Index of Multiple Deprivation (SIMD) decile and type of death. Logistic regression models were fitted using type of death as the outcome of interest and change in SIMD decile as the exposure of interest.

Results of 47012 deaths, 1458 were alcohol-related. Upward and downward mobility was observed for both types of death. An estimated 31 more deaths than expected were classified 'alcohol-related' among cases whose deprivation score decreased, while 204 more deaths than expected were classified 'alcohol-related' among cases whose initial deprivation ranking was in the four most deprived deciles. Becoming more deprived and first deprivation category were both associated with increased odds of type of death being alcohol-related after adjusting for confounders.

Conclusion This study suggests that health selection appears to contribute less to the deprivation gradient in alcohol-related mortality in Scotland than an individual's initial area deprivation category.

\section{INTRODUCTION}

Despite overall improvements in population health, health inequalities remain a stubborn problem in developed countries. ${ }^{1} 2$ A greater understanding of the mechanisms that create health inequalities is vital for designing effective policies and interventions. ${ }^{3}$ The Black Report hypothesised four mechanisms of health inequalities: artefact, health selection, materialist and cultural/behavioural. ${ }^{4}$ The materialist explanation, which identifies socioeconomic position (SEP) as the main causal pathway for health inequalities, emerged as the dominant theory following a polarised debate during the 1980s opposed by the reverse causation of health selection theory, where poor health status causes loss of SEP. $^{5}{ }^{6}$ Current consensus is that while health selection theory is a plausible mechanism in health inequalities for specific conditions or population groups, it is likely that it contributes only a small amount to the overall gradient of health inequality. 378

One cause of death for which health selection has been presented as a plausible mechanism is alcohol-related mortality. ${ }^{9-11}$ In particular, Jones and Sumnall's conceptual framework provides a useful visualisation of the hypothesised pathways between SEP and problem alcohol use (p. 10). ${ }^{9}$ Alcohol-related mortality is an outcome measure for the Scottish Government's national alcohol strategy ${ }^{12}$ and for the long-term monitoring of health inequalities in Scotland. ${ }^{13}$ However, the appropriateness of alcohol mortality as a measure of health inequalities has been questioned due to the possibility of reverse causation, citing 'chronic alcoholism [as] the quintessential example' of conditions which are likely lead to downwards socioeconomic mobility prior to death (p. 664). ${ }^{10}$

A lack of published evidence has been noted for reverse causation in terms of the effects of alcohol use on poverty. ${ }^{9}$ Two Scottish cohort studies have investigated the potential for health selection in relation to alcohol-related harm. One concluded that '[d]rinking patterns could both influence and be influenced by social mobility' (p. 336), ${ }^{14}$ while the other 'found little support for reverse causation' (p. 1). ${ }^{15}$ These previous studies focused on alcohol-related health harms as an outcome and used survey data which can be prone to selection bias.

By contrast, this study used routine administrative data to investigate whether individuals who died in Scotland from an alcohol-related condition showed greater movement into more deprived categories of area-based deprivation compared with individuals who died from other causes. In turn, this allowed us to assess whether alcohol-related mortality by area deprivation category at time of death is a suitable measure for monitoring health inequalities.

\section{METHODOLOGY}

Deaths recorded in Scotland during 2013 among people aged 21 years and over $(n=51860)$ were 
extracted from Scotland's national dataset for deaths held by the National Records of Scotland (NRS). Deaths were coded by type ('alcohol-related' or 'other' using the International Classification of Diseases 10 codes included in the NRS's definition of alcohol-related deaths ('F10', 'G312', 'G621', 'I426', 'K292', 'K70', 'K73', 'K740', 'K741', 'K742', 'K746', 'K860', 'X45', 'X65', 'Y15'). ${ }^{16}$ Area deprivation decile was assigned at death using the Scottish Index of Multiple Deprivation (SIMD). The SIMD provides relative deprivation ranking for small area geographies (2001-based data zones). ${ }^{17}$ The most recent version of SIMD used in this study (SIMD2012), combines 38 indicators across seven domains: income, employment, health, education, skills and training, housing, geographic access and crime. The weighted sum of the seven domain scores are then ranked from most deprived (rank 1) to least deprived (rank 6505). For our study, SIMD is presented as population-weighted deciles.

Deaths were linked to Scottish acute hospital records (SMR01) from 1996 onwards using the Community Health Index population register. Routine data linkage between the NRS and SMR01 datasets allowed the provision of earlier SIMD deciles, matched on the relevant SIMD version for the year of admission. Data completeness was high with over $96 \%$ of cases linked with previous SMR01 records $(n=50041)$. Cases with fewer than 365 days between first recorded SIMD and death were excluded from the analyses as this was felt to be an insufficient length of time for social drift to have occurred ( $n=4661 ; 9 \%)$.

Descriptive statistics were calculated to compare the proportion of cases that increased, decreased or showed no change from earliest recorded deprivation decile to decile at time of death. $\chi^{2}$ tests were calculated to estimate the difference between observed and expected alcohol-related deaths by first SIMD and type of death. Logistic regression models were built using type of death as the outcome of interest and change in SIMD decile as the exposure of interest. Age group, sex, first recorded SIMD decile and number of days from first recorded SIMD measure to death were included as covariates within the model. All statistical analyses were undertaken in SPSS V.19.

Ethical approval was not required for this study as these data are routinely linked by NHS National Services Scotland's Information Services Division. Confidential data release procedures were followed to allow data to be shared with colleagues at NHS Health Scotland for analysis.

\section{RESULTS}

From a total of 47012 deaths included in the study, 1458 $(3.1 \%)$ were alcohol-related. In $31.0 \%$ of alcohol-related and $28.8 \%$ of other deaths, SIMD decile had decreased (indicating downward mobility) between first recorded decile and decile at death (figure 1). Forty-six per cent of cases for both types of death showed no change in decile, while $22.6 \%$ of alcohol-related and $24.5 \%$ of other deaths increased decile. Association between type of death and change in decile from first recorded hospital discharge to death was found to be statistically significant (linear-by-linear association $(\mathrm{df}=1, \mathrm{n}=47012)=4.595$, $\mathrm{P}=0.03$ ) but very weak (Kendall's tau-c $=-0.004$ ). Based on comparison of observed and expected counts produced from $\chi^{2}$ tests, an estimated 31 more deaths than expected were classified as alcohol-related among cases whose deprivation score decreased, while 204 more deaths than expected were classified as alcohol-related among cases whose initial deprivation ranking was in the four most deprived deciles.

The median number of days between first SIMD record and death was 3518 for alcohol-related deaths and 3823 for other deaths, suggesting that it is appropriate to adjust for time between first record and death in the logistic regression model.

Logistic regression Model 1 investigated type of death as the outcome of interest and first recorded SIMD decile as the exposure of interest $(C=0.848)$ (table 1$)$. First recorded SIMD deciles 3-10 were associated with decreasing odds of type of death being alcohol-related, compared with first recorded SIMD decile 1 (most deprived) while adjusting for covariates. The addition of change in SIMD decile as the exposure of interest in Model 2 marginally improved the model $(\mathrm{C}=0.851)$. Decreased SIMD decile was associated with a significant increase to the odds of type of death being alcohol-related, compared with no change in SIMD decile while adjusting for covariates. Increased SIMD decile was not significantly associated with type of death.

\section{DISCUSSION}

This study suggests that while persons who die from alcohol-related conditions are more likely to experience social drift than those who die from other causes, health selection appears to contribute less to the deprivation gradient in alcohol-related mortality in Scotland than initial area deprivation category. The contribution of downward social mobility was unlikely to be

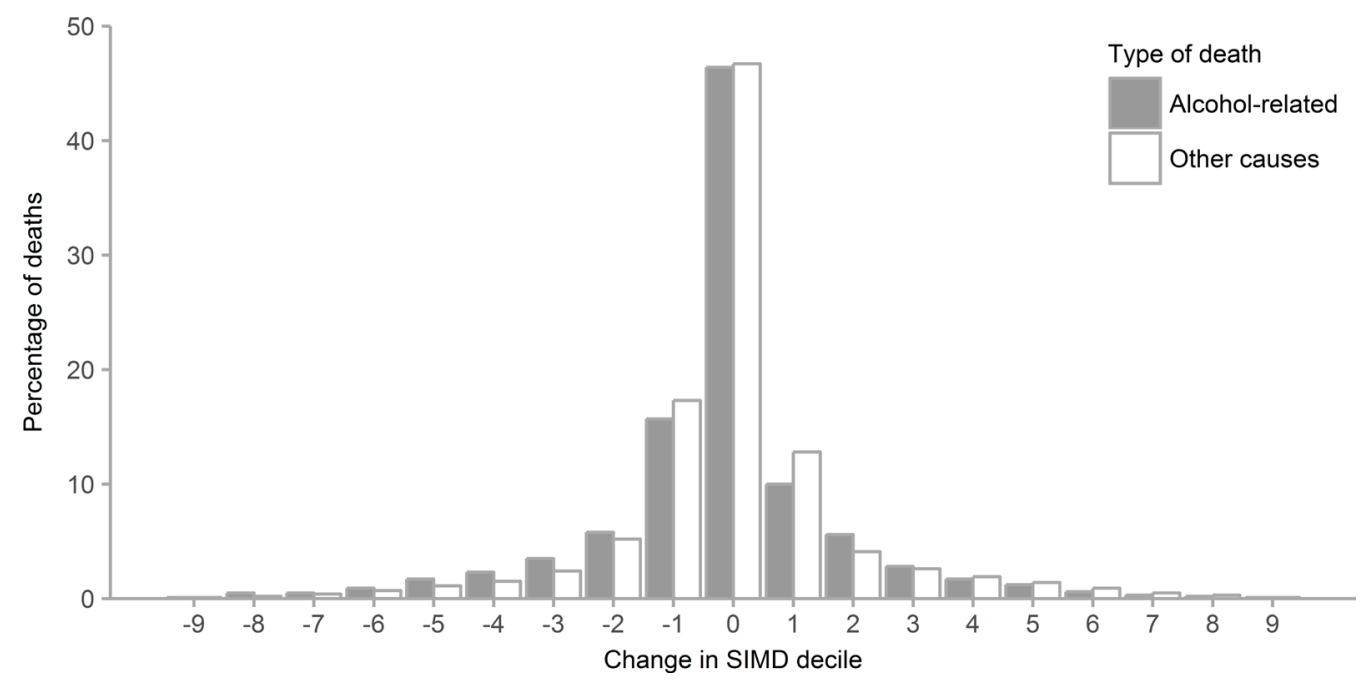

Figure 1 Change in SIMD decile from first SMR01 record to death, by type of death. SIMD, Scottish Index of Multiple Deprivation. 
Table 1 Adjusted ORs for alcohol-related death versus other type of death

\begin{tabular}{|c|c|c|c|c|c|c|c|c|}
\hline & \multicolumn{4}{|c|}{ Model 1} & \multicolumn{4}{|c|}{ Model 2} \\
\hline & \multirow[t]{2}{*}{$\operatorname{Exp}(B)$} & \multirow[t]{2}{*}{$\mathbf{P}$} & \multicolumn{2}{|c|}{$95 \% \mathrm{Cl}$ for $\operatorname{Exp}(\mathrm{B})$} & \multirow[t]{2}{*}{$\operatorname{Exp}(B)$} & \multirow[t]{2}{*}{$\mathbf{P}$} & \multicolumn{2}{|c|}{$95 \% \mathrm{Cl}$ for $\operatorname{Exp}(\mathrm{B})$} \\
\hline & & & Lower & Upper & & & Lower & Upper \\
\hline \multicolumn{9}{|c|}{ Age (baseline: 21-30years) } \\
\hline $31-40$ years & 3.60 & $<0.01$ & 1.88 & 6.89 & 3.55 & $<0.01$ & 1.85 & 6.81 \\
\hline $41-50$ years & 5.62 & $<0.01$ & 3.02 & 10.44 & 5.70 & $<0.01$ & 3.07 & 10.60 \\
\hline $51-60$ years & 3.83 & $<0.01$ & 2.07 & 7.08 & 3.96 & $<0.01$ & 2.14 & 7.32 \\
\hline $61-70$ years & 1.77 & 0.07 & 0.96 & 3.27 & 1.84 & 0.05 & 1.00 & 3.40 \\
\hline $71-80$ years & 0.54 & 0.05 & 0.29 & 1.00 & 0.56 & 0.07 & 0.30 & 1.04 \\
\hline 81 years and over & 0.11 & $<0.01$ & 0.05 & 0.20 & 0.11 & $<0.01$ & 0.06 & 0.21 \\
\hline Sex (baseline: male) & 0.51 & $<0.01$ & 0.45 & 0.57 & 0.51 & $<0.01$ & 0.45 & 0.58 \\
\hline \multicolumn{9}{|c|}{ Days from 1st SIMD to death (baseline: $366-1154$ days) } \\
\hline $1155-1859$ days & 1.15 & 0.23 & 0.92 & 1.44 & 1.09 & 0.47 & 0.86 & 1.37 \\
\hline $1860-2508$ days & 1.19 & 0.14 & 0.94 & 1.49 & 1.09 & 0.45 & 0.87 & 1.38 \\
\hline 2509-3174 days & 1.33 & 0.01 & 1.07 & 1.67 & 1.21 & 0.10 & 0.96 & 1.53 \\
\hline 3175-3813 days & 1.00 & 0.99 & 0.78 & 1.27 & 0.90 & 0.39 & 0.70 & 1.15 \\
\hline 3814-4444 days & 1.12 & 0.36 & 0.88 & 1.41 & 0.99 & 0.91 & 0.77 & 1.26 \\
\hline 4445-5119 days & 1.10 & 0.43 & 0.87 & 1.39 & 0.97 & 0.82 & 0.76 & 1.24 \\
\hline $5120-5884$ days & 0.97 & 0.80 & 0.76 & 1.24 & 0.85 & 0.20 & 0.66 & 1.09 \\
\hline 5885-6184 days & 1.01 & 0.95 & 0.80 & 1.28 & 0.89 & 0.33 & 0.69 & 1.13 \\
\hline $6185-6568$ days & 0.87 & 0.25 & 0.68 & 1.11 & 0.77 & 0.04 & 0.59 & 0.99 \\
\hline \multicolumn{9}{|c|}{ First recorded SIMD decile (baseline=most deprived decile) } \\
\hline SIMD decile 2 & 0.83 & 0.05 & 0.69 & 1.00 & 0.76 & $<0.01$ & 0.63 & 0.91 \\
\hline SIMD decile 3 & 0.73 & $<0.01$ & 0.60 & 0.88 & 0.64 & $<0.01$ & 0.53 & 0.79 \\
\hline SIMD decile 4 & 0.81 & 0.03 & 0.66 & 0.98 & 0.70 & $<0.01$ & 0.57 & 0.86 \\
\hline SIMD decile 5 & 0.75 & 0.01 & 0.61 & 0.92 & 0.64 & $<0.01$ & 0.51 & 0.79 \\
\hline SIMD decile 6 & 0.60 & $<0.01$ & 0.48 & 0.76 & 0.52 & $<0.01$ & 0.41 & 0.66 \\
\hline SIMD decile 7 & 0.66 & $<0.01$ & 0.52 & 0.83 & 0.56 & $<0.01$ & 0.44 & 0.71 \\
\hline SIMD decile 8 & 0.55 & $<0.01$ & 0.42 & 0.72 & 0.47 & $<0.01$ & 0.36 & 0.61 \\
\hline SIMD decile 9 & 0.63 & $<0.01$ & 0.49 & 0.82 & 0.53 & $<0.01$ & 0.41 & 0.70 \\
\hline Least deprived decile & 0.56 & $<0.01$ & 0.42 & 0.74 & 0.48 & $<0.01$ & 0.36 & 0.64 \\
\hline \multicolumn{9}{|c|}{ Change in SIMD decile (baseline=no change) } \\
\hline Decrease & - & - & - & - & 1.46 & $<0.01$ & 1.27 & 1.69 \\
\hline Increase & - & - & - & - & 1.04 & 0.64 & 0.90 & 1.20 \\
\hline
\end{tabular}

SIMD, Scottish Index of Multiple Deprivation.

due to chance and amounted to 31 more deaths than expected being classified as alcohol-related. Comparison of unadjusted observed and expected counts from $\chi^{2}$ tests and examination of adjusted coefficients from the final logistic regression model would suggest that initial deprivation category is more strongly associated with type of death being classed as alcohol-related. The findings therefore support the monitoring of inequalities in alcohol-related mortality by area-based measures of deprivation, particularly as these are routinely available within administrative datasets and are likely to be less sensitive to social drift than individual measures of SEP. ${ }^{11}$

This study used a large sample drawn from routinely collected population datasets with high level of coverage and completeness to investigate change in area-based deprivation classification prior to alcohol-related mortality. As such, it is relevant to the monitoring of health inequalities in Scotland and other countries. This study also has a number of limitations. First, as change in area-based deprivation decile is based on an individual's first recorded hospital admission, any health selection effect prior to this could not be identified. Second, the relative nature of SIMD ranking means that individuals could change decile following the publication of a new iteration without physically moving residence. Third, measures of alcohol consumption are not available within these administrative datasets, though another study produced similar findings even when accounting for differences in consumption. ${ }^{15}$ Fourth, it is plausible that individual measures of SEP such as income might be more sensitive to social drift effects. ${ }^{11}$ Limitations to area-based measures including the ecological fallacy can lead to the misidentification of individuals at risk. ${ }^{18}$ However, as area-based deprivation measures are commonly used to target public health interventions and are used for national monitoring of health inequalities in Scotland, these were of particular interest in the present study.

Previous research has indicated that materialist explanations and health selection are likely to contribute to inequalities in alcohol-related mortality. ${ }^{9} 1114$ However, as with the wider debate regarding the causes of health inequalities, this does not mean that the two explanations are equally evidenced or exert the same level of effect. ${ }^{915}$ This relationship is likely to be further complicated by the 'alcohol harm paradox' whereby similar 
levels of self-reported alcohol consumption are associated with greater alcohol-related harm in areas of greater deprivation. ${ }^{15} 19$ It is plausible that the alcohol harm paradox could mitigate or exacerbate health selection and further research would be useful to investigate the alcohol harm paradox's potential roles in relation to health selection.

This study provides a novel and useful analysis of area-based inequalities in alcohol-related mortality and the potential underlying explanations. It is unlikely that a simple causal pathway exists for alcohol-related mortality where harmful alcohol consumption is randomly distributed in the population and is followed by a substantial drift towards lower SEP prior to death. Based on this study and other published evidence, it appears that while materialist accounts appear to be the dominant explanation for health inequalities, an increasingly nuanced health selection account could provide a complementary explanation of how disadvantage might accumulate over the life course and across generations. ${ }^{6} 11$

\section{What is already known on this subject}

- Although widely discounted as a major cause of health inequalities, health selection has been presented as a plausible theory explaining the patterning of alcohol-related mortality by area deprivation.

- There is relatively little published evidence regarding the contribution of health selection to alcohol-related harms.

\section{What this study adds}

- This study does not support the hypothesis that health selection is the dominant mechanism that explains the deprivation gradient in alcohol-related mortality in Scotland.

- This study supports the use of area deprivation as a useful measure of the impact of socioeconomic status causing alcohol-related mortality.

Contributors Study design and critical input into manuscript: $A P, R G, L G, J L, G M$, MR. Analyses of data: AP, RG, JL. Preparation of manuscript: AP.

Competing interests GM advises the Scottish Government on the indicators for their 'Long-term Monitoring report on Health Inequalities'. MR is a current member, and GM, LG and RG previous members, of the project team responsible for monitoring and evaluating Scotland's alcohol strategy.

Provenance and peer review Not commissioned; externally peer reviewed.

Open Access This is an Open Access article distributed in accordance with the Creative Commons Attribution Non Commercial (CC BY-NC 4.0) license, which permits others to distribute, remix, adapt, build upon this work non-commercially, and license their derivative works on different terms, provided the original work is properly cited and the use is non-commercial. See: http://creativecommons.org/ licenses/by-nc/4.0/

(C) Article author(s) (or their employer(s) unless otherwise stated in the text of the article) 2018. All rights reserved. No commercial use is permitted unless otherwise expressly granted.

\section{REFERENCES}

1 Smith K. Beyond evidence-based policy in public health - the interplay of ideas. Basingstoke: Palgrave McMillan, 2013:60-1.

2 Mackenbach JP. The persistence of health inequalities in modern welfare states: the explanation of a paradox. Soc Sci Med 2012;75:761-9.

3 McCartney G, Collins C, Mackenzie M. What (or who) causes health inequalities: theories, evidence and implications? Health Policy 2013;113:221-7.

4 Department of Health \& Social Security. Inequalities in health. In: Townsend P, Whitehead M, Davidson N, eds. The Black Report. London: Penguin Books, 1992:104-15.

5 Macintyre S. The black report and beyond: what are the issues? Soc Sci Med 1997:44:723-45

6 West P. Rethinking the health selection explanation for health inequalities. Soc Sci Med 1991;32:373-84.

7 Blane D, Smith GD, Bartley M. Social selection: what does it contribute to social class differences in health? Sociology of Health and IIIness 1993;15:1-15.

8 UCL Institute of Health Equity. Review of social determinants and the health divide in the WHO european region: final report. Copenhagen: World Health Organization, 2013:5-12.

9 Jones L, Sumnall H. Understanding the relationship between poverty and alcohol misuse. Liverpool: Liverpool John Moores University, 2016:9-17.

10 Frank J, Haw S. Best practice guidelines for monitoring socioeconomic inequalities in health status: lessons from Scotland. Milbank Q 2011;89:658-93.

11 Mäkelä P. Alcohol-related mortality as a function of socio-economic status. Addiction 1999;94:867-86.

12 Beeston C, McAdams R, Craig N, et al. Monitoring and evaluating Scotland's alcohol strategy - final report. Edinburgh: NHS Health Scotland, 2016:18-21.

13 Scottish Government. Long-term monitoring of health inequalities. Edinburgh: Scottish Government, 2015:36-8.

14 Hart CL, Davey Smith G, Upton MN, et al. Alcohol consumption behaviours and social mobility in men and women of the midspan family study. Alcohol Alcohol 2009;44:332-6.

15 Katikireddi SV, Whitley E, Lewsey J, et al. Socioeconomic status as an effect modifier of alcohol consumption and harm: analysis of linked cohort data. Lancet Public Health 2017;2:e267-e276

16 National Records of Scotland. Alcohol-related deaths in Scotland, 1979 to 2015. Edinburgh: National Records of Scotland, 2016. https://www.nrscotland.gov.uk/ statistics-and-data/statistics/statistics-by-theme/vital-events/deaths/alcohol-relateddeaths

17 The Scottish Government. The Scottish index of multiple deprivation. http://www.gov. scot/Topics/Statistics/SIMD

18 Fischbacher C. 2014. Identifying "deprived individuals": are there better alternatives to the Scottish Index of Multiple Deprivation (SIMD) for socioeconomic targeting in individually based programmes addressing health inequalities in Scotland? Glasgow: Scottish Public Health Observatory.

19 Bellis MA, Hughes K, Nicholls J, et al. The alcohol harm paradox: using a national survey to explore how alcohol may disproportionately impact health in deprived individuals. BMC Public Health 2016;16:111. 